\title{
Hierarchical Cobalt Selenides as Highly Efficient Microwave Absorbers with Tunable Frequency Response
}

Min Zeng ${ }^{a *}$, Qian Cao $^{a}$, Jue liu $^{a *}$, Baiyu Guo ${ }^{a}$, Xiaozhu, Hao ${ }^{a}$, Qingwei Liu ${ }^{a}$, Xiaofang Liu ${ }^{a}$, Xin Sun $^{a}$, Xixiang Zhang ${ }^{b}$, Ronghai Yu*

${ }^{a}$ School of Materials Science and Engineering, Beihang University, Beijing 100191, China.

${ }^{b}$ King Abdullah Univ Sci \& Technol, Div Phys Sci \& Engn, Thuwal 239556900, Saudi Arabia

ABSTRACT: Microwave absorbing materials have attracted much attention in solving electromagnetic interference and pollution problems. Hierarchical cobalt selenides have been obtained through a facile selenization annealing process. The as-prepared samples exhibit distinct reflection losses $\left(R_{L}\right)$ and frequency responses via tailoring their crystalline configurations, with excellent absorption in $\mathrm{Ku}, \mathrm{X}$, or $\mathrm{C}$ band. All the samples show $R_{L}$ greater than or near to $-50 \mathrm{~dB}$ with effective bandwidths more than $4 \mathrm{GHz}$, indicating they may serve as high-efficient and frequency-tunable microwave absorbers. Especially, the sample annealed at $400{ }^{\circ} \mathrm{C}$ shows a competitive $R_{L}$ of $-62.04 \mathrm{~dB}$ at $9.92 \mathrm{GHz}$ with a thickness of $2.25 \mathrm{~mm}$; meanwhile, its effective absorption bandwidth reaches $5.36 \mathrm{GHz}$ with a thickness as small as $1.56 \mathrm{~mm}$. The cobalt selenides as microwave absorbers exhibit promising prospect applied in complex electromagnetic environment.

KEYWORDS: cobalt selenides, crystalline configuration, tunable frequency absorption, microwave absorber, reflection loss 


\section{INTRODUCTION}

In recent years, electromagnetic interference and pollution are becoming more and more serious, which may cause malfunction in sensitive electronic devices and even hazard people's health ${ }^{1}$. Using a microwave absorber, which interacts with the wave and converts its energy into other forms, is an effective way to diminish the harmful radiation ${ }^{2-4}$. Recently, extensive studies on the microwave absorbers have promoted the research of the absorbing materials and the development of shielding technologies. These absorbers are generally composed of magnetic or dielectric fillers ${ }^{3,5}$. The main absorbing mechanism of dielectric materials is dielectric loss $^{9}$, while magnetic loss dominates the absorbing performance of the magnetic materials ${ }^{10,11}$.

Reflection loss $\left(R_{L}\right)$ and effective absorption bandwidth are the two important parameters for microwave absorbers. Meanwhile, when considering the applications in complex electromagnetic environments, adjustment of the absorption frequency is particularly significant. Although lots of materials with good absorbing properties have been found, those that possess both good and tunable performance are still limited and need to be further discovered. Tremendous amount of efforts have been made toward transition metal sulfides and selenides, including $\mathrm{MoS}_{2}{ }^{9}, \mathrm{MoSe}_{2}{ }^{12}, \mathrm{CuS}^{13}, \mathrm{Co}_{\mathrm{x}} \mathrm{S}_{\mathrm{y}}{ }^{14}, \mathrm{Ni}_{\mathrm{x}} \mathrm{S}_{\mathrm{y}}{ }^{15}, \mathrm{NixCo}_{3-\mathrm{x}} \mathrm{S}_{4}{ }^{16}$, and $\mathrm{ZnS}^{17}$, as fillers in the microwave absorbers composites due to their intrinsic electric conductivity, which can induce electronic polarization, conductive loss, and dielectric relaxation under an alternated electromagnetic field ${ }^{18}$. The cobalt selenides, a typical kind of transition metal selenides, exhibit controllable electric properties by adjusting stoichiometric compositions and crystalline structures. Moreover, they have lattice similarity with cobalt sulfides, which have been reported to demonstrate good responses to electromagnetic wave ${ }^{14}$, possibly leading to unique microwave 
absorption performance ${ }^{19,20}$. As far as we know, although cobalt selenides have been applied in other fields such as advanced energy storage systems ${ }^{21}$, dye sensitized solar cell ${ }^{22}$, and hydrogen evolution $^{23}$, there are rarely reports on their applications in the microwave absorption area. In order to reveal the potentiality, this paper aims at exploring the microwave absorption properties of cobalt selenides. The relationship between the crystalline structure and $R_{L}$ makes the study intriguing because we also try to synthesize the microwave absorbing media with tunable frequency responds.

Here, hierarchical cobalt selenides have been prepared by a hydrothermal method, followed by a selenization process. The cobalt selenides obtained under different temperatures show very good microwave absorption performance. The crystalline structures and electromagnetic properties are tunable by regulating the selenization temperature, carving a facile approach to tune the effective absorbing frequency and capacity.

\section{EXPERIMENTAL SECTION}

\subsection{Materials}

Cobalt chloride hexahydrate $\left[\mathrm{CoCl}_{2} \cdot 6 \mathrm{H}_{2} \mathrm{O}, \geq 99.0 \%\right]$ and urea $\left(\mathrm{H}_{2} \mathrm{NCONH}_{2}, \geq 99.0 \%\right)$ were purchased from Sinopharm Chemical Reagent Co., Ltd. (Shanghai, China). Ascorbic acid $\left[\mathrm{C}_{6} \mathrm{H}_{8} \mathrm{O}_{6}, \geq 99.7 \%\right]$ was purchased from Xilong Scientific Co., Ltd. (Guangdong, China). Selenium powder (Se, 99\%) was purchased from Aladdin Biochemical Technology Co., Ltd. (Shanghai, China). Ethanol $\left(\mathrm{C}_{2} \mathrm{H}_{5} \mathrm{OH}, \geq 99.7 \%\right)$ was supplied by Beijing Chemical Company (Beijing, China). All the reagents were used as received without any further purification.

\subsection{Synthesis}


$0.951 \mathrm{~g} \mathrm{CoCl}_{2} \cdot 6 \mathrm{H}_{2} \mathrm{O}, 3 \mathrm{~g} \mathrm{C}_{6} \mathrm{H}_{8} \mathrm{O}_{6}$, and $3.6 \mathrm{~g} \mathrm{CO}\left(\mathrm{NH}_{2}\right)_{2}$ were added into $240 \mathrm{~mL}$ deionized water under stirring to obtain a homogeneous solution. Then the mixture was transferred into a Teflon autoclave and heated at $160{ }^{\circ} \mathrm{C}$ for $6 \mathrm{~h}$ in an electric oven. After natural cooling, the obtained precursor was collected, washed with ethanol and deionized water for several times, and dried. Finally, the precursor was heat treated with Se (mass ratio 1:2) in $\mathrm{N}_{2}$ atmosphere at $300{ }^{\circ} \mathrm{C} / 400{ }^{\circ} \mathrm{C} / 500{ }^{\circ} \mathrm{C} / 600{ }^{\circ} \mathrm{C}$ for $2 \mathrm{~h}$ with a ramping rate of $2{ }^{\circ} \mathrm{C} \cdot \mathrm{min}^{-1}$, as shown in scheme 1 , yielding the as-prepared products denoted as S-300, S-400, S-500, and S-600, respectively. In addition, cobalt oxide was also prepared for comparison with precursor annealed in air at $400{ }^{\circ} \mathrm{C}$ for $2 \mathrm{~h}$.

\subsection{Characterization}

The crystalline structures were acquired by X-ray diffraction (XRD, D/MAX-2500, Rigaku). The thermogravimetric (TGA) and differential scanning calorimetry (DSC) analyses of the precursor with Se powder (mass ratio 1:2) were investigated using a TGA/DSC simultaneous thermal analyzer (Netzsch STA449F3). The surface morphologies of the samples were observed with a scanning electron microscopy (SEM, JSM 7500, JEOL). Transmission electron microscopy (TEM, TF30, FEI) with the attached energy-dispersive X-ray (EDX) spectroscopy was used to estimate the microstructures and elemental distribution. The valance information was obtained with an X-ray photoelectron spectroscopy (XPS, Axis Supra, Kratos). The asprepared powders were compressed into small bars for conductivity measurement by a fourelectrode probe method with a YAOS Resistivity Tester. Static magnetic properties at room temperature were detected by a vibrating sample magnetometer (VSM, 7404, Lake Shore). The complex permittivities and complex permeabilities of the samples were tested on a vector network analyzer (VNA, Agilent N5234A) within 2-18 GHz, using the powder mixed with 
paraffin wax (mass ratio 1:1) and pressed into circle rings in a mold with an outer diameter of 7 $\mathrm{mm}$ and an inner diameter of $3 \mathrm{~mm}$.

\section{RESULTS AND DISCUSSION}

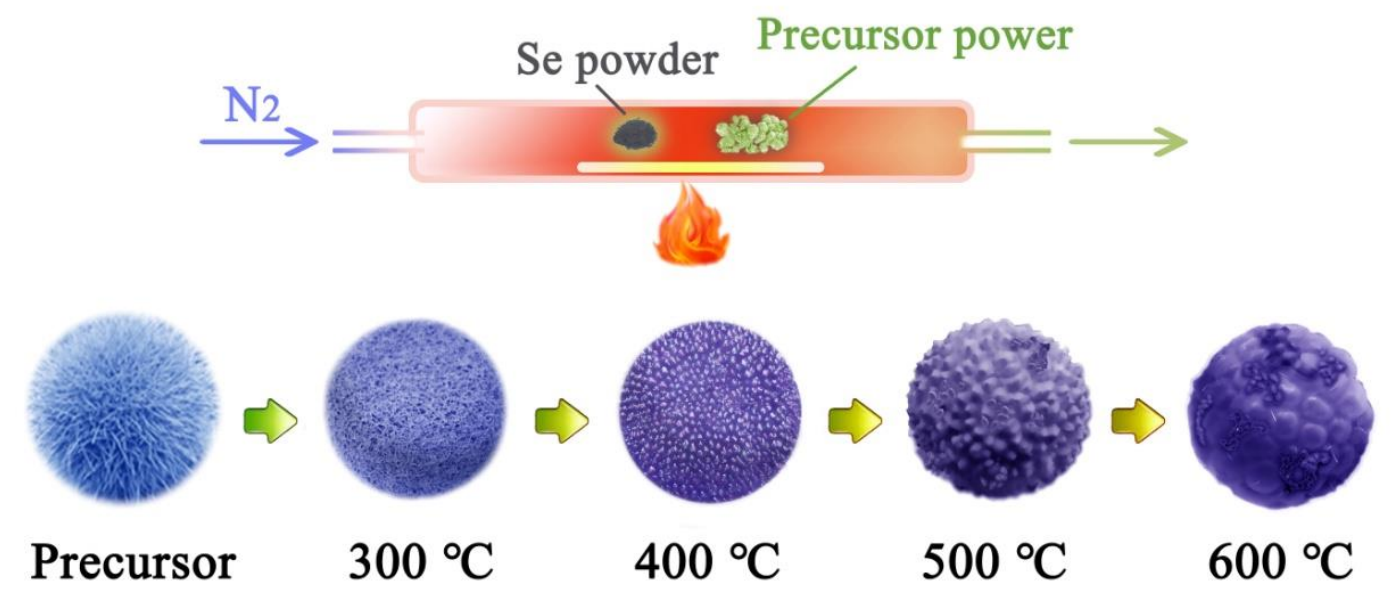

Scheme 1. Schematic diagram of selenization process and evolution of surface morphology at different annealed temperatures.

The XRD pattern of the precursor shown in Figure $\mathrm{S} 1$ corresponds to rhombohedral $\mathrm{CoCO}_{3}$ (JCPDS no. 78-0209). The weak peaks illustrate that the precursor has poor crystallinity. Figure 1 shows the XRD patterns of the cobalt selenides calcined under different temperatures. Better crystallinity is observed in the sample with increasing selenization temperature. S-300 has obvious diffraction peaks at $34.5^{\circ}, 36.0^{\circ}, 47.7^{\circ}$, and $50.2^{\circ}$, which corresponds to (111), (120), (211), and (002) crystal planes of orthorhombic $\mathrm{CoSe}_{2}$ (JCPDS no. 53-0449). This crystal structure is also obtained in the literature with $300{ }^{\circ} \mathrm{C}$ selenization ${ }^{24}$. S-400 and S-500 have extra peaks at $37.6^{\circ}, 51.7^{\circ}, 58.9^{\circ}$, and $76.1^{\circ}$. This is owing to the appearance of cubic structured $\mathrm{CoSe}_{2}$ (JCPDS no. 89-2002), showing that they are composed of orthorhombic and cubic $\mathrm{CoSe}_{2}$. It is observed that the characteristic peaks of cubic $\mathrm{CoSe}_{2}$ rise and peaks of orthorhombic $\mathrm{CoSe}_{2}$ 
decline in S-500, proving the increase of cubic $\mathrm{CoSe}_{2}$ content. When further increasing the temperature, except for characteristic peaks of orthorhombic and cubic $\mathrm{CoSe}_{2}$, peaks at $33.2^{\circ}$, $33.8^{\circ}, 44.8^{\circ}$, and $50.5^{\circ}$ index well to (101), (002), (102), and (110) planes, respectively, of hexagonal CoSe (JCPDS no. 89-2004), indicating more complex structure in S-600 25 .
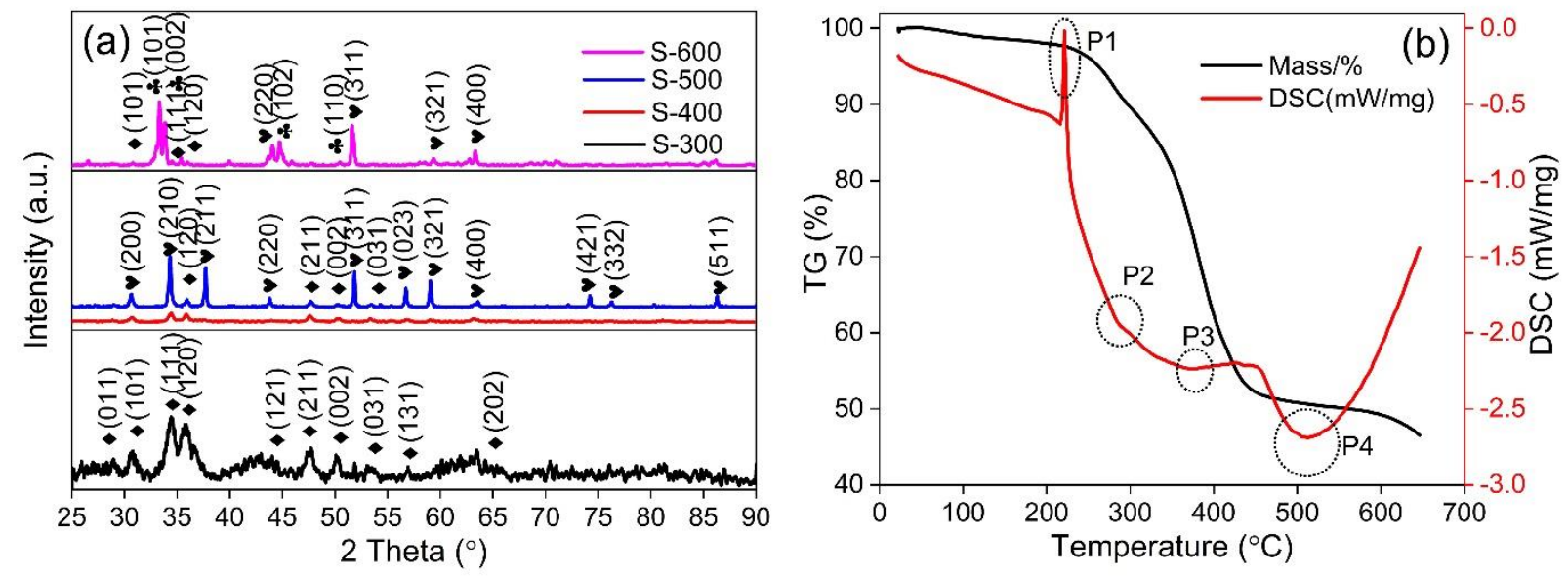

Figure 1. (a) XRD patterns of the samples: S-300, S-400, S-500, and S-600. ( $\bullet, \bullet$, and represent orthorhombic $\mathrm{CoSe}_{2}$ (JCPDS no. 53-0449), cubic structured $\mathrm{CoSe}_{2}$ (JCPDS no. 892002), and hexagonal CoSe (JCPDS no. 89-2004), respectively; (b) TGA/DSC curves of the precursor and Se powder mixture.

The TGA/DSC results of the precursor and Se powder mixture in $\mathrm{N}_{2}$ atmosphere are shown in Figure 1(b). The sharp endothermic peak P1 at $222{ }^{\circ} \mathrm{C}$ corresponds to the selenium melting ${ }^{25}$. Then the precursor begins to react with Se to form $\mathrm{CoSe}_{2}$, showing a small peak P2 at $286{ }^{\circ} \mathrm{C}$. From this test we can estimate that it is reasonable to obtain $\mathrm{CoSe}_{2}$ when annealing at $300{ }^{\circ} \mathrm{C}$, which is also proved by the XRD pattern of S-300. Peak P3 at $375^{\circ} \mathrm{C}$ suggests that crystal phase transformation happens ${ }^{26}$, which is in accordance with cubic $\mathrm{CoSe}_{2}$ appearance at $400{ }^{\circ} \mathrm{C}$ selenization. Further increasing the selenization temperature to $512{ }^{\circ} \mathrm{C}$, Peak P4 emerges, 
illustrating that some $\mathrm{CoSe}_{2}$ transforms to $\mathrm{CoSe}$, confirmed by $\mathrm{CoSe}$ existence under $600{ }^{\circ} \mathrm{C}$ selenization. Meanwhile, the gradual weight loss in the selenization process could be explained by the precursor decomposition and Se evaporation.

The precursor sphere (shown in Figure S2) has a fluffy surface and an average size of about $1 \mu \mathrm{m}$. After selenization in different temperatures, the morphologies of the obtained samples are shown in Figure 2. $\mathrm{CoCO}_{3}$ decomposition and selenium diffusion happen mildly at lower temperature. As a result, the S-300 sphere in Figure 2(a) inherits the morphology from the precursor. In S-400 (Figure 2(b)), the decomposition and selenization are much tense. Although most of the spheres still retain the topographical status of the precursor, the small crystalline grains in the spheres begin to grow into bigger irregular-shaped ones, which corresponds to phase change and recrystallization during annealing process as suggested by $\mathrm{XRD}^{27}$. When treated in $500{ }^{\circ} \mathrm{C}$, apparently two different grains can be observed on the surface, indicating more furious recrystallization happens (Figure 2(c)). When the selenization temperature reaches $600{ }^{\circ} \mathrm{C}$, much bigger crystalline grains can be observed, leaving S-600 much bumpier surface (Figure 2(d)). This morphological and structural conversion can be explained by the strain and atom reorganization ${ }^{28-30}$.

Typically, the microwave absorption of sub-micron powder material is highly correlated to the surface morphology besides the diameter and the intrinsic electromagnetic property. The hierarchical structures of the particles tend to scatter the incident wave and induce multiple reflections $^{5,12,31}$. Meanwhile, the complex structure is conducive to the excitation of surface and interfacial polarization, thus improving dielectric loss and endowing the absorbers strong microwave absorption. 


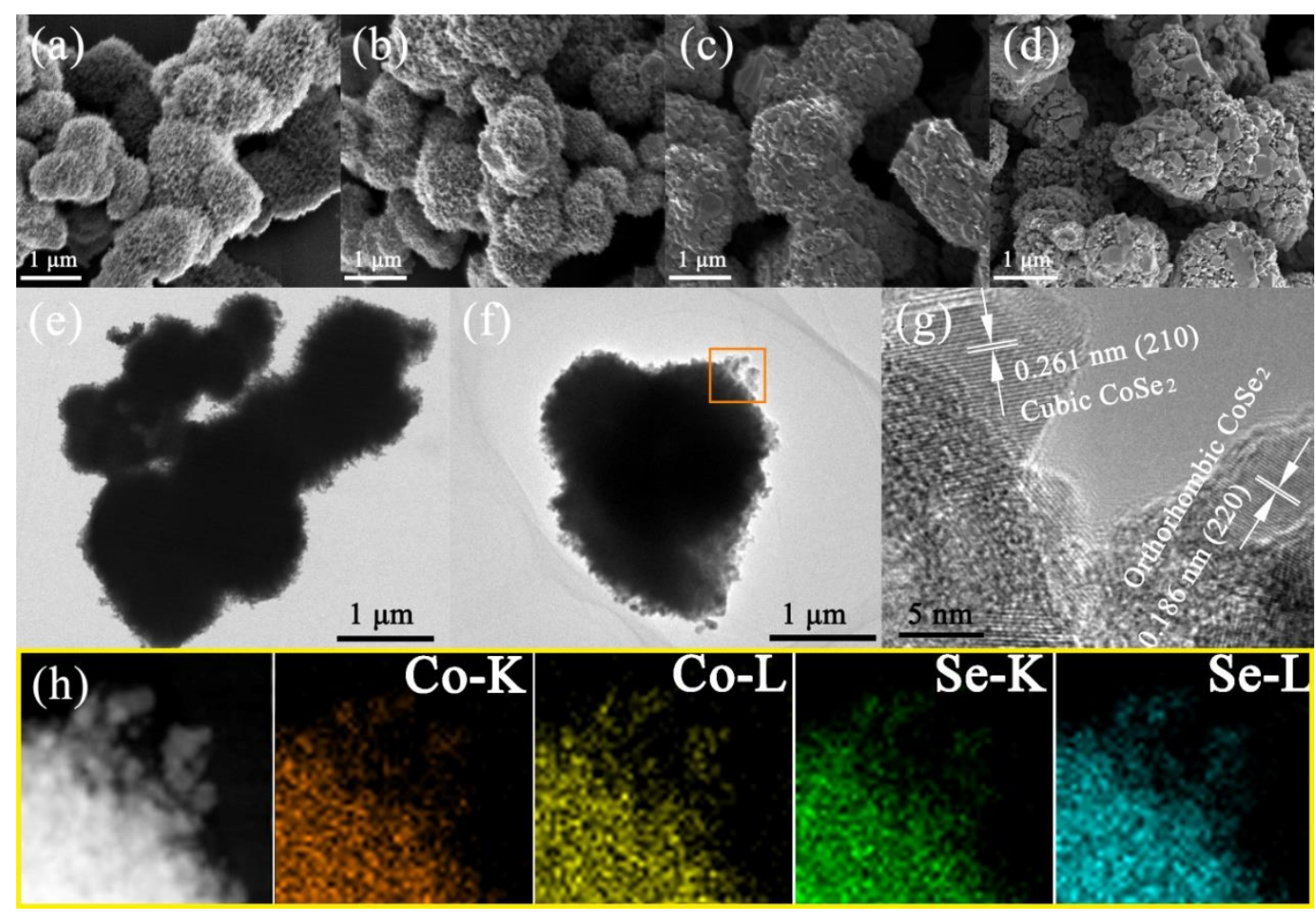

Figure 2. SEM images of (a) S-300, (b) S-400, (c) S-500, (d) S-600 and (e, f) TEM images, (g) HRTEM image, (h) elemental mapping of S-400.

Here, S-400 is selected for further microstructure analysis. The hierarchical surface can be confirmed by the TEM images in Figure 2(e, f). The HRTEM image (Figure 2(g)) shows that the lattice fringes of $0.261 \mathrm{~nm}$ and $0.186 \mathrm{~nm}$ correspond to the (210) plane of cubic $\mathrm{CoSe}_{2}$ and (220) plane of orthorhombic $\mathrm{CoSe}_{2}$, respectively, which is in accordance with the XRD results. Meanwhile, the EDX mapping in Figure 2(h) demonstrates the homogeneous distribution of Co and Se in the sample. 

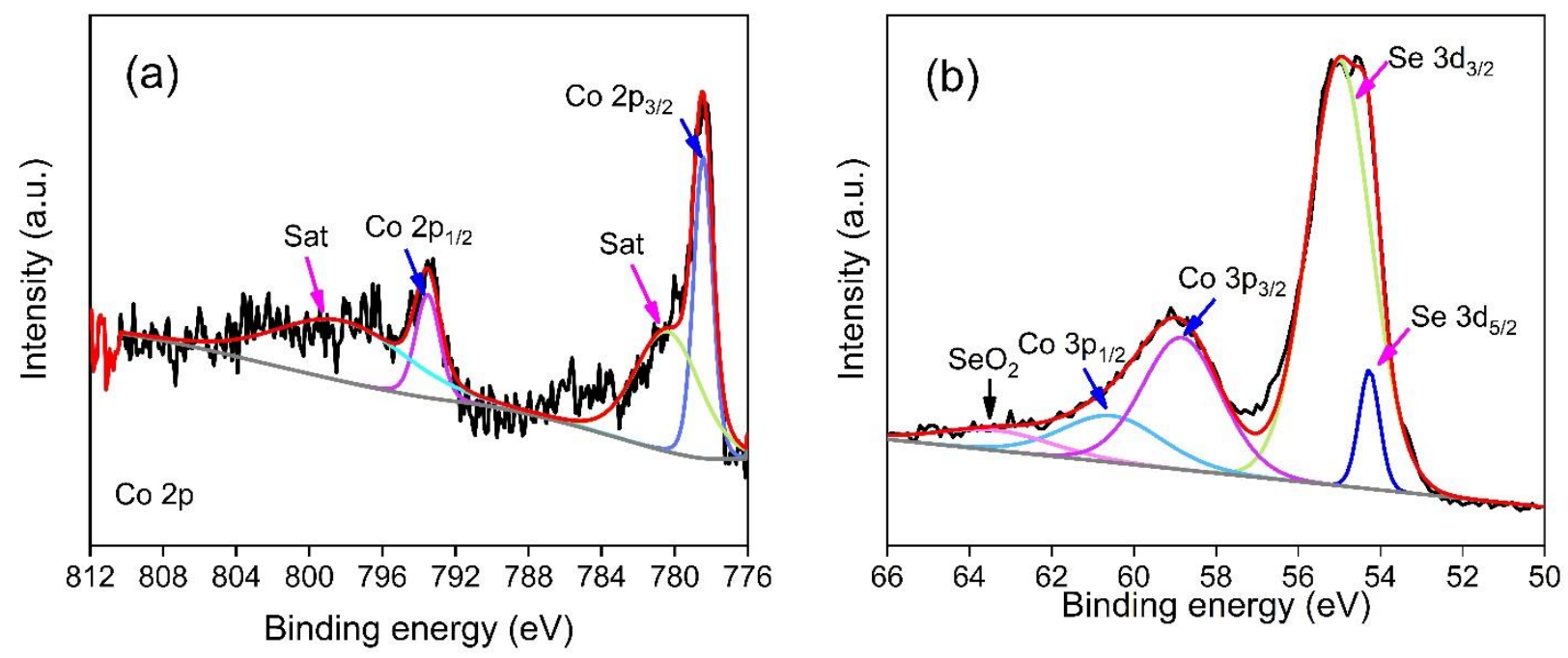

Figure 3. XPS spectra of S-400: (a) Co 2p; and (b) Se 3d.

XPS can be adopted to analyze the surface valence state information. S-400 is selected as a typical double-phase composite and the corresponding data are presented in Figure 3. Four peaks are observed in Co 2p spectrum (Figure 3(a)), among which two main peaks at $778.4 \mathrm{eV}$ and $793.5 \mathrm{eV}$ are attributed to Co $2 \mathrm{p}_{3 / 2}$ and Co $2 \mathrm{p}_{1 / 2}$, respectively, proving the existence of $\mathrm{Co}^{2+}$ in $\mathrm{CoSe}_{2}$. Another two peaks located at $780.4 \mathrm{eV}$ and $798.2 \mathrm{eV}$ are the satellite peaks ${ }^{32}$. Figure 3(b) shows the Se $3 d$ spectrum, in which the $\mathrm{Se} 3 \mathrm{~d}_{5 / 2}$ and $\mathrm{Se} 3 \mathrm{~d}_{3 / 2}$ peaks located at $54.3 \mathrm{eV}$ and 55.0 $\mathrm{eV}$, respectively, correspond to $\mathrm{Se}^{-}$in $\mathrm{CoSe}_{2}$. And peaks at $58.8 \mathrm{eV}$ and $60.5 \mathrm{eV}$ are attributed to Co $3 p_{3 / 2}$ and Co $3 p_{1 / 2}$, respectively. Another broad peak at $59.1 \mathrm{eV}$ is ascribed to Se-O bond, suggesting the presence of $\mathrm{SeO}_{2}$ owing to the sample's air exposure ${ }^{33},{ }^{34}$. Overall, the Se spectrum is in accordance with the interaction between Co and Se.

Complex permittivity $\left(\varepsilon_{r}=\varepsilon^{\prime}-j \varepsilon^{\prime \prime}\right)$ and permeability $\left(\mu_{r}=\mu^{\prime}-j \mu^{\prime \prime}\right)$ are the two very important parameters to evaluate the microwave absorbing performance. Figure $4(a, b)$ show the real and imaginary parts of the complex permittivity. $\varepsilon^{\prime}$ value of S-300 decreases from 5.75 to 
4.20 smoothly, and the corresponding $\varepsilon^{\prime \prime}$ value shows the decrease tendency from 1.49 to 0.59 with slight variations at 2-18 GHz. For S-400, the values of $\varepsilon^{\prime}$ and $\varepsilon^{\prime \prime}$ are significantly enhanced and the curves fluctuate strongly with frequency compared with that of S-300. It is noted that $\varepsilon^{\prime}$ fluctuates from 13.31 and finally down to 8.73 and $\varepsilon^{\prime \prime}$ fluctuates dramatically with enhancement from 2.23 to 5.81 in the range of 2-18 GHz. Such concavo-convex $\varepsilon^{\prime \prime}$ curve suggests strong dissipation towards the incident microwave energy ${ }^{35}$. In the curves of S-500, $\varepsilon^{\prime}$ value begins from 18.05 and ends at 11.70 , while $\varepsilon^{\prime \prime}$ is from 3.65 and up to 5.93 , showing bigger values than that of S-400. However, S-600 fails to present larger $\varepsilon^{\prime}$ and $\varepsilon^{\prime \prime}$ values compared with S-500. Its $\varepsilon^{\prime}$ is close to that of S-400 before $15 \mathrm{GHz}$ and rises a little bit up to 11 at $18 \mathrm{GHz}$, while its $\varepsilon^{\prime \prime}$ is smaller than that of S-400, with a median of about 2.50 . The variation trend of complex permittivity of S-600 differentiates from those of S-300, S-400 and S-500, which may be ascribed to the appearance of CoSe crystalline structure, arousing distinct dielectric properties.

The dielectric losses $\left(\tan \delta \mathrm{e}=\varepsilon^{\prime \prime} / \varepsilon^{\prime}\right)$ of different samples are displayed in Figure 4(c). The variations in the dielectric losses show distinguishingly frequency-dependent trends within $2-18$ GHz. In S band, S-300 is the best. In C band, S-400 has the highest values. In X band, S-600 peaks around $9 \mathrm{GHz}$. In $\mathrm{K}_{\mathrm{u}}$ band, S-400 tips up again with its summit around $17 \mathrm{GHz}$. Since dielectric loss associates with the absorbing capability of a dielectric absorber, those composites may be selectively used as highly efficient absorbers and satisfy high-performance absorption in a tunable frequency range. 

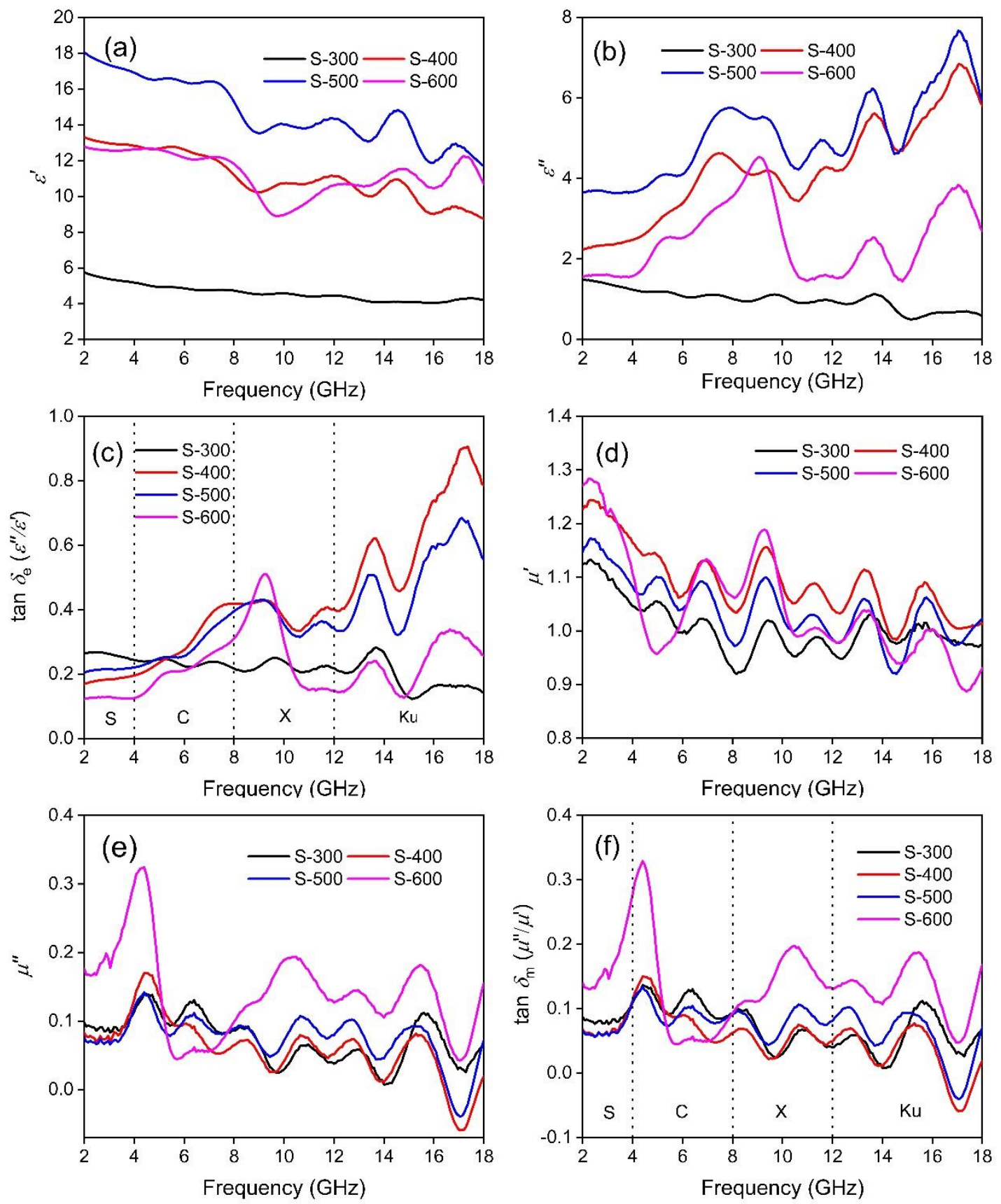

Figure 4. Frequency dependent of (a) real parts and (b) imaginary parts of complex permittivity; (c) dielectric losses; (d) real parts and (e) imaginary parts of complex permeability; (f) magnetic losses of the samples. 
The origin of dielectric loss can be explored from the complex permittivity in some extent. Generally, consumption of the dynamic field energy stems from conductivity loss and polarization loss ${ }^{36}$. The conductivity loss is relative to the electric conductivity of the absorbers, and polarization loss origins from electronic polarization, ionic polarization, dipole orientation polarization, and interfacial polarization ${ }^{8,37}$. Given that the first two modes generally happen in terahertz and petahertz frequencies rather than in gigahertz region, only the dipole polarization and interfacial polarization are the probable reasons that generate Debye relaxations and shape the complex permittivity charicteristics ${ }^{38}$. Therefore, the roles of polarization relaxation in attenuating microwave energy are analyzed based on the Debye theory ${ }^{39,40}$, whose equation is illustrated:

$$
\left(\varepsilon^{\prime}-\frac{\varepsilon_{s}+\varepsilon_{\infty}}{2}\right)^{2}+\left(\varepsilon^{\prime \prime}\right)^{2}=\left(\frac{\varepsilon_{s}-\varepsilon_{\infty}}{2}\right)^{2}
$$

where $\varepsilon_{s}$ represents the relative permittivity under static, $\varepsilon_{\infty}$ represents the relative permittivity at the high frequency limit.

Figure S3 shows the Cole-Cole curves obtained through plotting $\varepsilon^{\prime}$ versus $\varepsilon^{\prime \prime}$. A Cole-Cole semicircle on the curve is regarded as a specific Debye relaxation process ${ }^{41}$. It shows that all the samples display multiple distorted polarization relaxations, which is in accordance with multiple peaks in $\varepsilon^{\prime \prime}$ curves. These multiple polarization relaxations can be induced by both interfacial polarization and dipole polarization. The crystal interface, interface between cobalt selenides and paraffin wax, and hierarchical structure of the samples can help generate strong interfacial polarization. As observed in complex permittivity, S-300 containing pure orthorhombic $\mathrm{CoSe}_{2}$ has the lowest $\varepsilon^{\prime}$ and $\varepsilon^{\prime \prime}$ values. S-400 and S-500, who are the mixtures of orthorhombic and cubic $\mathrm{CoSe}_{2}$, present larger $\varepsilon^{\prime}$ and $\varepsilon^{\prime \prime}$ values owing to the heterogeneous crystal interfaces. 
However, S-600 fails to possess increasing $\varepsilon^{\prime}$ and $\varepsilon^{\prime \prime}$ values. Meanwhile, the interfaces between orthorhombic and cubic $\mathrm{CoSe}_{2}$ reduce and $\mathrm{CoSe}$ and $\mathrm{CoSe}_{2}$ interfaces increase, which are identified with XRD results. It is deduced that this component lowers the polarization effect when compared to S-400 and S-500. In terms of dipole polarization, the electric dipoles are generated due to the different electronegativity of $\mathrm{Co}$ and $\mathrm{Se}$, contributing to the dissipation of electromagnetic wave.

Since the Cole-Cole semicircles are severely distorted, other mechanism, including conductive loss, contribute to the dielectric loss. The electric conductivities of S-300, S-400, S500, and S-600 listed in Table S1 are $6.83 \times 10^{-6}, 4.65 \times 10^{2}, 8.73 \times 10^{2}$, and $9.29 \times 10^{2} \mathrm{~S} / \mathrm{m}$, respectively. The conductivities get larger with better crystallization degree of the samples. According to the free electron theory, higher conductivity will arouse stronger dielectric response in the alternating field ${ }^{42}$. The trend of $\varepsilon^{\prime \prime}$ satisfies the theory for S-300, S-400, and S500. However, the $\varepsilon^{\prime \prime}$ values of S-600 decline compared with that of S-500, which may due to the strong polarization loss dominating the dielectric behavior.

Complex permeability releases the information of magnetic loss for the materials ${ }^{43}$. The real part and imaginary part of the complex permeability correspond to the storage and loss capacities for magnetic energy, respectively ${ }^{44}$. Figure $4(\mathrm{~d}, \mathrm{e})$ show the frequency-dependent $\mu^{\prime}$ and $\mu^{\prime \prime}$ values of the samples. Roughly, the $\mu^{\prime}$ values of the samples fluctuate within $0.9-1.3$ as frequency increasing. Accordingly, the $\mu^{\prime \prime}$ curves for all the samples oscillate in the measured frequencies. It is noted that $\mu^{\prime \prime}$ shows negative values for S-400 and S-500 within $16-18 \mathrm{GHz}$. According to the Maxwell equations, the alternating electric field and magnetic field can be transformed into each other ${ }^{45}$. In this case, the dipole or interface resonance generates oscillating 
electric field, and this electric field consequently induces an additional alternating magnetic field superposing to the oscillating magnetic moments under high-frequency wave. The negative permeability will occur when the induced magnetic field is higher than the latter ${ }^{45-47}$. The static magnetic properties of the samples are presented in Figure S4. It shows that S-600 possesses more obvious ferromagnetism than the others. Its $\mu^{\prime \prime}$ values are higher and therefore induce stronger magnetic loss in most situations expect 5-8 GHz.

Magnetic tangent $\left(\tan \delta_{\mathrm{m}}=\mu^{\prime \prime} / \mu^{\prime}\right)$ related to the magnetic loss is calculated and shown in Figure 4(f), having a similar trend with the imaginary parts of the complex permeability. The values of S-300, S-400 and S-500 are around 0.1 , induced by their small $\mu$ " values. The values of S-600 are relatively higher than the others with a peak of 0.33 around $4.5 \mathrm{GHz}$ and several crests in $\mathrm{C}$ and $\mathrm{Ku}$ bands, indicating the magnetic loss makes appreciable contribution to the microwave attenuation. The magnetic loss mainly comes from hysteresis loss, domain wall resonance, eddy current effect, natural resonance and exchange resonance ${ }^{48,49}$. In the gigahertz range, magnetic hysteresis loss is negligible in the weak field ${ }^{50}$. Domain wall resonance is also ignorable for it occurs at a much lower frequency range ${ }^{45}$. Thus, only eddy current loss, natural resonance and exchange resonance need to be taken into consideration. If the factor $C_{0}=$ $\mu^{\prime \prime}\left(\mu^{\prime}\right)^{-2} f^{-1}$ ( $f$ is the frequency) keeps constant, the eddy current loss will be the sole reason for magnetic $\operatorname{loss}^{51,52}$. As shown in Figure S5, $C_{0}$ for all the samples displays frequent dependence, suggesting the contribution comes from natural resonance and exchange resonance besides eddy current loss. In theory, the natural resonance usually occurs at lower frequency. However, hierarchical structures can help to shift the resonance frequency to higher range $\mathrm{e}^{44}$. As a result, the steep peak in the $\mu^{\prime \prime}$ curve between $2-6 \mathrm{GHz}$ may benefit from the natural resonance. The following peaks in higher frequencies are attributed to the exchange resonance ${ }^{52,53}$. 
Base on the electromagnetic wave transmission theory, the intensity of the microwave absorption is expressed by $R_{L}$ employing relative complex permittivity $\left(\varepsilon_{r}=\varepsilon^{\prime}-j \varepsilon^{\prime \prime}\right)$ and complex permeability $\left(\mu_{r}=\mu^{\prime}-j \mu^{\prime \prime}\right)$ with the given layer thickness under a specific frequency. It can be deduced from the following equations ${ }^{54,55}$ :

$$
\begin{gathered}
Z_{0}=\left(\frac{\mu_{0}}{\varepsilon_{0}}\right)^{1 / 2} \\
Z_{\text {in }}=\left(\mu_{r} / \varepsilon_{r}\right)^{1 / 2} \tanh \left(j \frac{2 \pi f t}{c}\right)\left(\mu_{r} \varepsilon_{r}\right)^{1 / 2} \\
R_{L}=20 \log \left|\frac{Z_{\text {in }}-Z_{0}}{Z_{\text {in }}+Z_{0}}\right|
\end{gathered}
$$

where $Z_{\text {in }}$ refers to the input impedance; $Z_{0}$ is the impedance of free space $\left(Z_{0}=377 \Omega\right)$. $\varepsilon_{0}$ and $\mu_{0}$ are the permittivity and permeability of the free space, respectively. $f$ is the frequency. $t$ is the thickness of the layer. $c$ is the velocity of light. Practicably, $R_{L}$ less than $-10 \mathrm{~dB}$ denotes a qualified absorption, which means more than $90 \%$ microwave is absorbed.

The $R_{L}$ maps versus $f$ and $t$ as two variables are shown in Figure 5(a-d). S-300 has an optimum $R_{L}$ value of $-50.93 \mathrm{~dB}$ at $16.48 \mathrm{GHz}$ in $\mathrm{Ku}$ band with a thickness of $6.82 \mathrm{~mm}$, and the effective bandwidth $\left(R_{L} \leq-10 \mathrm{~dB}\right)$ reaches a maximum value of $4.24 \mathrm{GHz}$ with a thickness of $6.79 \mathrm{~mm}$. The best microwave absorption performance is achieved by S-400, with an optimum $R_{L}$ value of $-62.04 \mathrm{~dB}(9.92 \mathrm{GHz}$ in $\mathrm{X}$ band, $2.25 \mathrm{~mm})$ and broad absorption bandwidth of 5.36 $\mathrm{GHz}$ with a small thickness of $1.56 \mathrm{~mm}$. For S-500, the optimum $R_{L}$ value and absorption bandwidth reach $-55.26 \mathrm{~dB}(5.12 \mathrm{GHz}, \mathrm{C}$ band, $3.46 \mathrm{~mm})$ and $4.72 \mathrm{GHz}(1.35 \mathrm{~mm})$, respectively. The $R_{L}$ and absorption bandwidth of S-600 terminate at $-47.28 \mathrm{~dB}(10.16 \mathrm{GHz}, \mathrm{X}$ band, $2.4 \mathrm{~mm})$ and $4.48 \mathrm{GHz}(1.5 \mathrm{~mm})$, respectively. Each sample shows the optimal $R_{L}$ values of about or 
higher than $-50 \mathrm{~dB}$ in different bands within 2-18 GHz. For comparison, the microwave absorption performance of cobalt oxide, which was obtained with air annealing instead of Se treatment, is also investigated. The optimal $R_{L}$ and bandwidth reach $-23.14 \mathrm{~dB}$ and $2.80 \mathrm{GHz}$ with a thickness of $7.5 \mathrm{~mm}$ as shown in Figure S6, which are much lower than the as-prepared cobalt selenides. To explore why, the data of the complex permittivity and complex permeability are analyzed. It is found that $\varepsilon^{\prime}$ and $\varepsilon^{\prime \prime}$ values (illustrated in Figure S7) are much lower than that of cobalt selenides. It has been reported that electrical conductivity is positively related to complex permittivity and one merit of transitional-metal selenides is high conductivity induced by higher electronic conductivity of the elemental selenium ${ }^{12}$. It is no wonder that cobalt selenide, as a dielectric type absorber, possess better microwave absorption performance than the corresponding cobalt oxide. Furthermore, the excellent microwave absorption performances are competitive with the reported cobalt-based materials and metal sulfides/selenides (Table 1), show these hierarchical cobalt selenides are promising candidates in microwave absorption.

$R_{L}$ and absorption bandwidth always relate to the thickness of the absorbers, as observed in Figure 5. Taking the pathway of microwave absorption into consideration, if the incident and reflected waves in the absorber are out of phase $\pi$, they will be destructive interference with each other. The thickness satisfied with the quarter-wavelength model is listed as the following ${ }^{56}$ :

$$
t_{m}=\frac{n c}{4 f \sqrt{\varepsilon_{r} \mu_{r}}}(n=1,3,5, \ldots)
$$

The $\lambda / 4$ matching geometric effect is shown in Figure 5(e-h). Two $R_{L}$ peaks, whose corresponding thicknesses equal to one-quarter and three-quarters of the wavelength, can be observed for S-300, while other three samples have only one sharp $R_{L}$ peak with the thickness satisfying one-quarter of wavelength. 

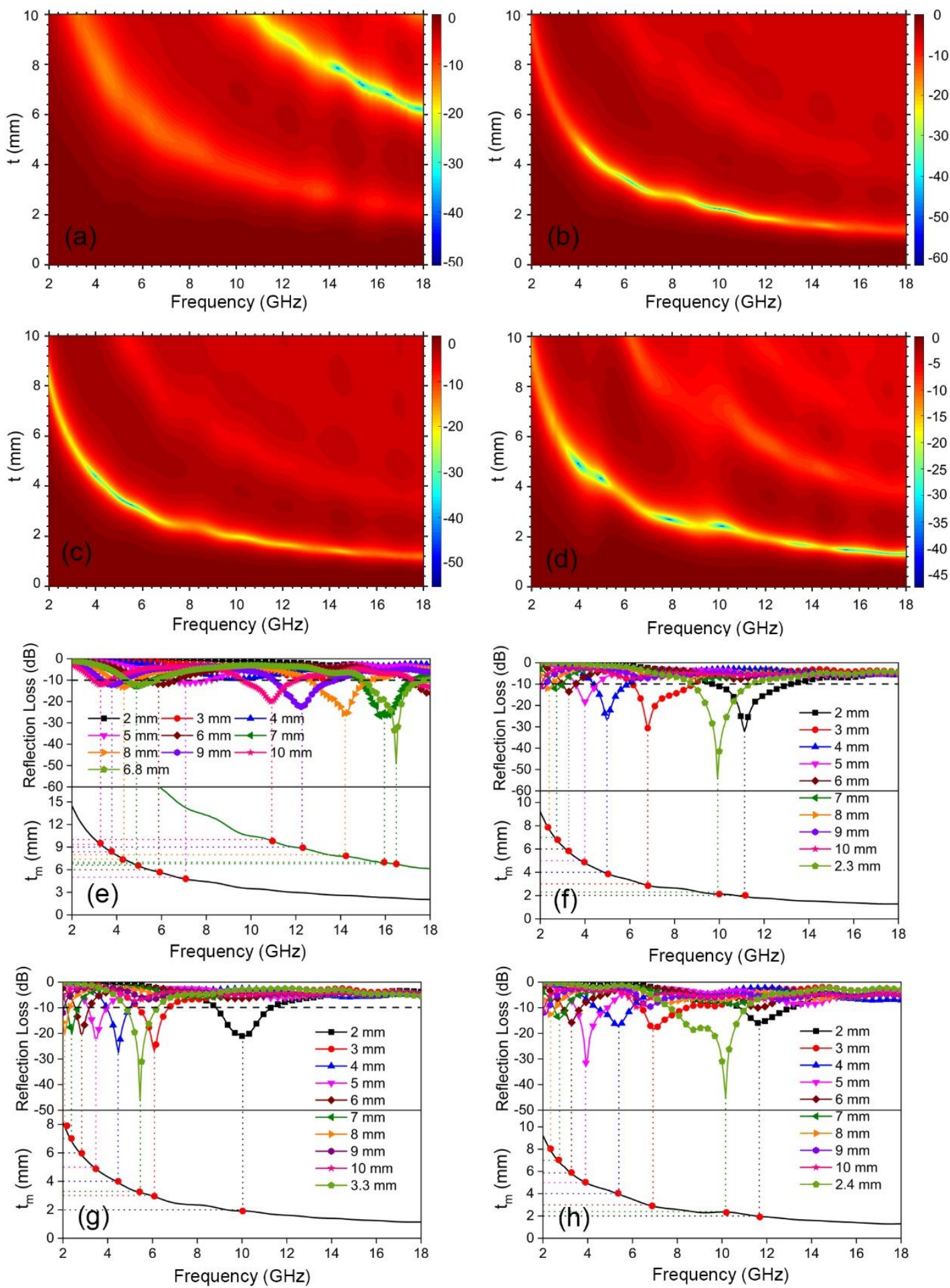

Figure 5. $R_{L}$ of (a) S-300, (b) S-400, (c) S-500, (d) S-600; $t_{m} v s$. $f$ at wavelengths of $\lambda / 4$ and $3 \lambda / 4$ for (e) S-300, (f) S-400, (g) S-500, and (h) S-600. 
So far, we have investigated the interactions between the microwave absorption performances and the morphologies, crystalline structures, dielectric properties, magnetic properties and geometric effects of the materials. The intuitional microwave dissipation mechanism still needs to be further discussed. Attenuation constant $\alpha$ and impedance matching $\left(\left|Z_{i n} / Z_{0}\right|\right)$ are the critical parameters.

On one hand, the attenuation constant $\alpha$, which is directly related to the $R_{L}$, satisfies the following equation ${ }^{57}$.

$$
\boldsymbol{\alpha}=\frac{\sqrt{2} \pi f}{c} \sqrt{\left(\mu^{\prime \prime} \varepsilon^{\prime \prime}-\mu^{\prime} \varepsilon^{\prime}\right)+\sqrt{\left(\mu^{\prime \prime} \varepsilon^{\prime \prime}-\mu^{\prime} \varepsilon^{\prime}\right)^{2}+\left(\mu^{\prime} \varepsilon^{\prime \prime}+\mu^{\prime \prime} \varepsilon^{\prime}\right)^{2}}}
$$

It is obvious that higher values of $\mu^{\prime \prime}$ and $\varepsilon^{\prime \prime}$ result in a larger $\alpha$. Meanwhile, $\alpha$ values almost keep increasing with elevated frequencies. As evidenced by Figure 6(a), $\alpha$ values for S-400, S500 and S-600 increase rapidly compared to that of S-300, suggesting stronger attenuation capabilities in the three samples.

On the other hand, the transmission behaviors of the microwave are greatly related to the impedance matching characteristics. Sufficient electromagnetic wave can propagate through an absorber and then be attenuated when there is a good impedance matching ${ }^{58,59}$. Therefore, when the value of impedance matching $\left(\left|Z_{i n} / Z_{0}\right|\right)$ is close to 1 that corresponds to zero-reflection at the interface, the best $R_{L}$ might occur. In Figure 6(b), the values of S-300 diverge from 1 except the cross point around $13 \mathrm{GHz}$. S-400 provides the values very close to 1 in $9-11 \mathrm{GHz}$. In the same region, S-500 also has the values next to 1 . For S-600, the values around 1 appear in the range of $11-13 \mathrm{GHz}$. 

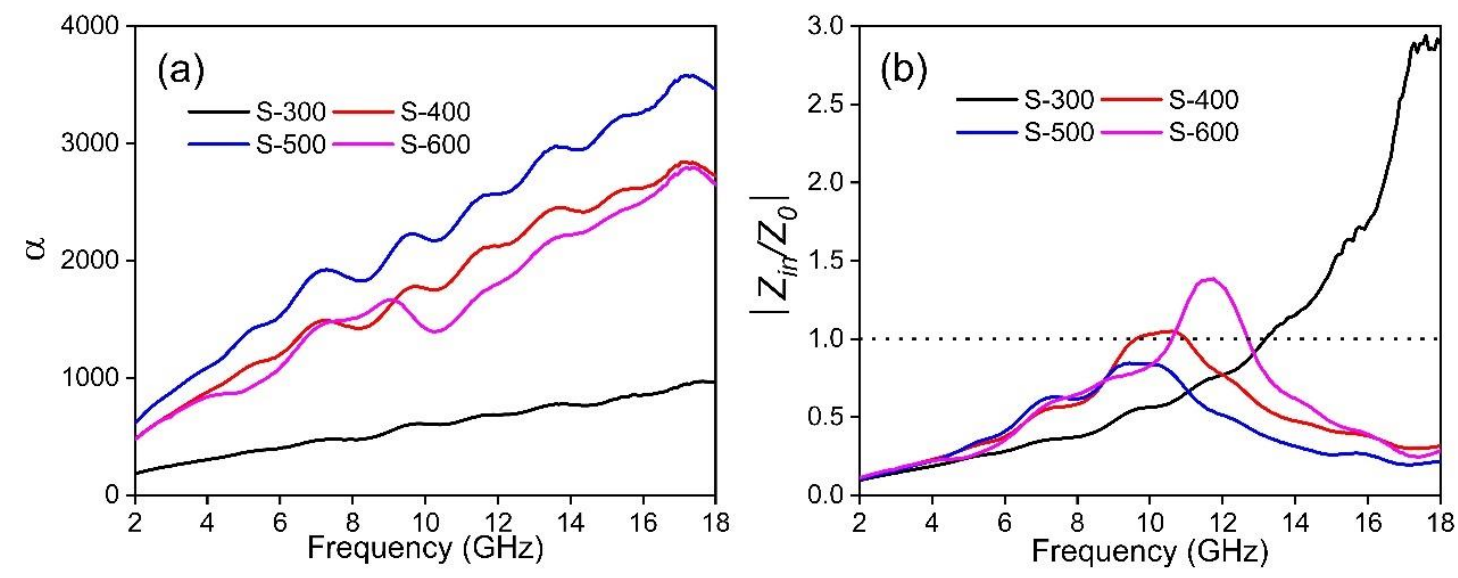

Figure 6. (a) attenuation constant $\alpha$, (b) impedance matching $\left(\left|Z_{i n} / Z_{0}\right|\right)$ of the samples.

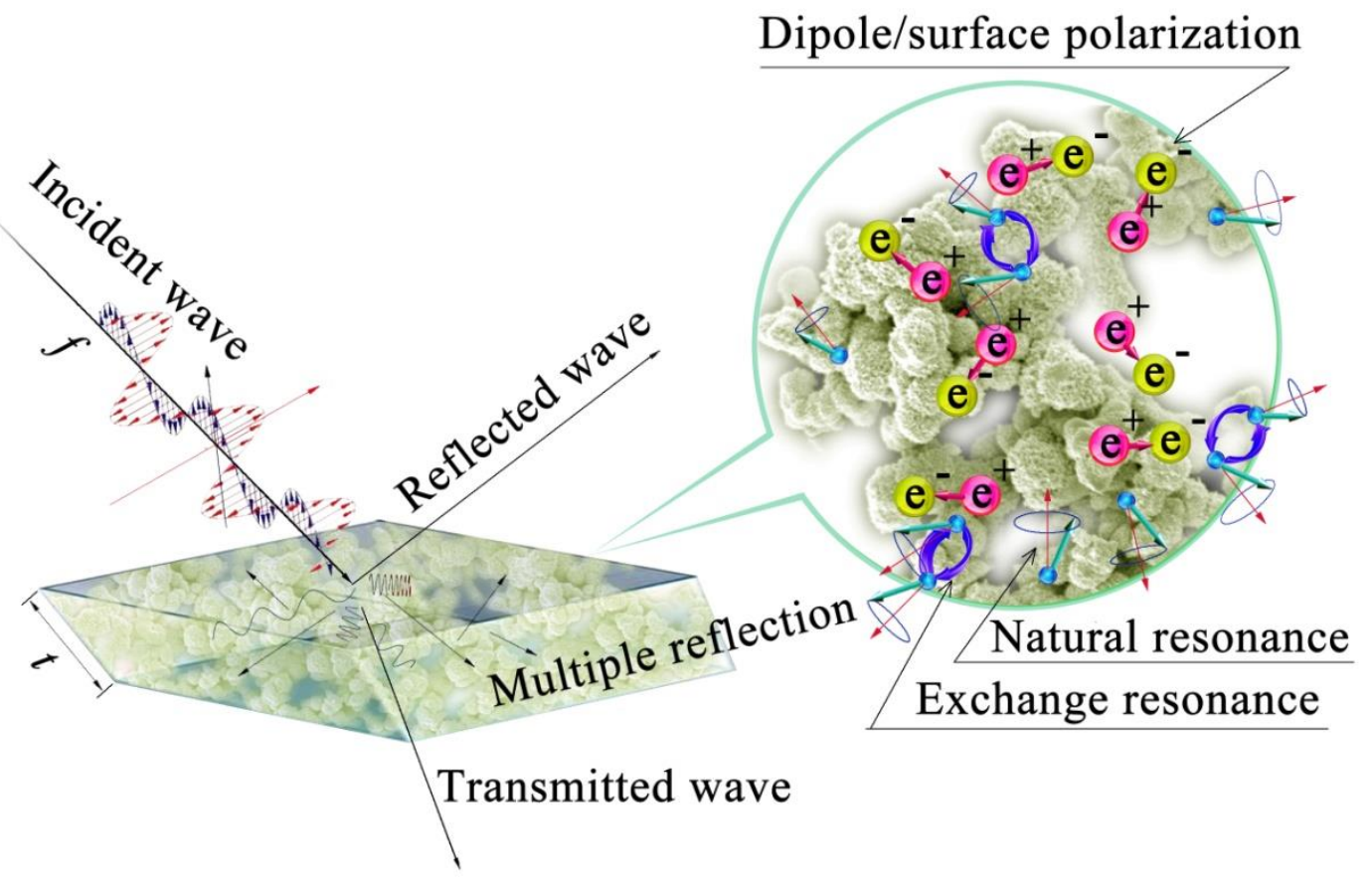

Scheme 2. Scheme of the microwave absorption process. 
Table 1. The microwave absorption properties of some metal sulfides/selenides and cobalt-based composites.

\begin{tabular}{|c|c|c|c|c|}
\hline $\begin{array}{l}\text { Electromagnetic } \\
\text { Absorbers }\end{array}$ & $\begin{array}{l}\text { Optimum } \quad \text { Reflection } \\
\text { Loss/dB (thickness/mm) }\end{array}$ & $\begin{array}{l}\text { Optimal } \\
\text { frequency/GHz }\end{array}$ & $\begin{array}{l}\text { Bandwidth/GH } \\
\text { z(thickness/mm } \\
\text { ) }\end{array}$ & References \\
\hline $\mathrm{CoS}$ & $-43.6(2)$ & 15.6 & $4.6(2)$ & [14] \\
\hline $\mathrm{Co}_{1-\mathrm{x}} \mathrm{S}$ & $-46.1(2.5)$ & 13.92 & $5.6(2.5)$ & [60] \\
\hline $\mathrm{Co}_{3} \mathrm{O}_{4} / \mathrm{RGO} / \mathrm{MDCF}$ & $-31.88(2.0)$ & 11.54 & $3.4(2.0)$ & [31] \\
\hline $\begin{array}{l}\text { carbon-based } \\
\mathrm{Co}(\mathrm{OH})_{2}-\mathrm{Co}_{3} \mathrm{O}_{4}\end{array}$ & $-55.8(2.03)$ & 10.56 & $3.6(2.03)$ & [61] \\
\hline $\mathrm{Co}_{3} \mathrm{O}_{4}$ & $-41.1(2.1)$ & - & $3.46(2.3)$ & {$[62]$} \\
\hline $\mathrm{Co} / \mathrm{CNTs}$ & $-49.16(2.5)$ & - & $4.2(2.5)$ & [63] \\
\hline $\mathrm{MoS}_{2}$ & $-54.72(3.5)$ & 9.1 & $4.2(3.5)$ & [64] \\
\hline $\mathrm{Co} / \mathrm{CoO}$ & $-21.7(2.3)$ & - & $6.1(2.3)$ & [65] \\
\hline $\mathrm{ZnS} / \mathrm{Ni}$ & $-42.4(2.2)$ & 12.3 & $4.3(2.2)$ & [66] \\
\hline $\mathrm{CuS} / \mathrm{ZnS}$ & $-22.6(3)$ & 9.7 & $2.4(3)$ & [67] \\
\hline $\mathrm{MoS}_{2}$ & $-38.42(2.4)$ & - & $4.1(2.4)$ & [9] \\
\hline S-300 & $-50.93(6.82)$ & 16.48 & $4.24(6.79)$ & This work \\
\hline S-400 & $-62.04(2.25)$ & 9.92 & $5.36(1.56)$ & This work \\
\hline S-500 & $-55.26(3.46)$ & 5.12 & $4.72(1.35)$ & This work \\
\hline S-600 & $-47.28(2.4)$ & 10.16 & $4.48(1.5)$ & This work \\
\hline
\end{tabular}

The microwave absorption mechanism of the samples is depicted in Scheme 2. When the incident wave reaches the surface of the absorber, part of the wave is reflected and transmitted, while the rest is absorbed inside the absorber. Complex mechanisms function to consume and converse the microwave energy. Firstly, multiple reflections induced by the abundant surface and 
interface prolong the transmission path, promoting the microwave attenuation. The hierarchical structures of cobalt selenides contribute to the multiple reflections. Secondly, the aggregation of polarized bound charges on the surface and junction of the complicated structures, along with the inside dielectric dipole polarization help generate relaxation in the altering electromagnetic field, which is beneficial for microwave energy consumption by scattering. Moreover, the natural resonance and exchange resonance behaviors exhaust the wave at the resonance frequencies. Finally, if the impedance matching is granted, the projected wave encountering its counterpart of opposite phase from the reflection will generate optimized absorption performance. Since the intrinsic electromagnetic properties are affected by the microstructure and valence state of the material, changing the crystalline structure and the surface morphology of the cobalt selenides via annealing will regulate the microwave absorbing performance. The hierarchical cobalt selenides are proved to be strong-absorption and broad-bandwidth microwave absorbers with tunable frequency response.

\section{CONCLUSION}

The hierarchical cobalt selenides have been successfully synthesized as promising microwave absorbers. Diverse crystalline structures and morphology configurations are regulated by a facile selenization treatment under different temperatures. Here, the as-prepared samples display competitive $R_{L}$ values and bandwidths with tunable frequency response. Specifically, S300 shows the optimal $R_{L}$ of $-50.93 \mathrm{~dB}$ in $\mathrm{Ku}$ band and an effective absorption bandwidth of 4.24 $\mathrm{GHz}$; The best $R_{L}$ and absorption bandwidth are $-62.04 \mathrm{~dB}(9.92 \mathrm{GHz}$ in $\mathrm{X}$ band, $2.25 \mathrm{~mm})$ and 5.36 GHz obtained from S-400. S-500 and S-600 also demonstrate excellent absorption performance $(-55.26 \mathrm{~dB}$ in $\mathrm{C}$ band, $3.46 \mathrm{~mm}$ and $-47.28 \mathrm{~dB}$ in $\mathrm{X}$ band, $2.40 \mathrm{~mm}$, respectively). 
Overall, by regulating and optimizing the microstructures, these hierarchical cobalt selenides are promising candidates for microwave absorption.

\section{ASSOCIATED CONTENT}

\section{Supporting Information}

XRD pattern and SEM image of the precursor; Cole-Cole curves, Conductivities of the asprepared cobalt selenides powders, VSM spectra, eddy current loss $C_{0}$ of the samples, $R_{L}$ of the cobalt oxide with different thicknesses, Frequency dependent complex permittivities, complex permeabilities of the cobalt oxide.

\section{AUTHOR INFORMATION}

\section{Corresponding Author}

*E-mail address: min zeng@buaa.edu.cn. Phone: +86-10-82317101.

*E-mail address: jueliu1992@163.com. Phone: +86-10-82317101.

*E-mail address: rhyu@buaa.edu.cn. Phone: +86-10-82317101.

\section{Author Contributions}

The manuscript was written through contributions of all authors. All authors have given approval to the final version of the manuscript.

\section{Funding Sources}

National Natural Science Foundation of China (51920105001, 51731002, U1832138, 51971008) and funding from State Key Lab of Advanced Metals and Materials (2019-Z08). 


\section{Notes}

The authors declare no competing financial interest.

\section{ACKNOWLEDGEMENTS}

This work was financially supported by National Natural Science Foundation of China (51920105001, 51731002, U1832138, 51971008) and State Key Lab of Advanced Metals and Materials (2019-Z08).

\section{REFERENCES}

1. Abdalla, I.; Salim, A.; Zhu, M.; Yu, J.; Li, Z.; Ding, B. Light and Flexible Composite Nanofibrous Membranes for High-Efficiency Electromagnetic Absorption in a Broad Frequency. ACS Appl. Mater. Interfaces 2018, 10 (51), 44561-44569.

2. He, G.; Duan, Y.; Pang, H.; Zhang, X. Rational Design of Mesoporous $\mathrm{MnO}_{2}$ Microwave Absorber with Tunable Microwave Frequency Response. Appl. Surf. Sci. 2019, 490, 372-382.

3. Ouyang, J.; Liu, T.; Yang, H.; Zhang, Y. Multiple Polarization Loss and Permittivity Adjusting of Halloysite/BN Co-doped Carbon/Cobalt Composites. J. Colloid. Interface Sci. 2019, 555, 509-518.

4. Liu, X.; Chen, Y.; Cui, X.; Zeng, M.; Yu, R.; Wang, G. S. Flexible Nanocomposites with Enhanced Microwave Absorption Properties based on $\mathrm{Fe}_{3} \mathrm{O}_{4} / \mathrm{SiO}_{2}$ Nanorods and Polyvinylidene Fluoride. J. Mater. Chem. A 2015, 3 (23), 12197-12204.

5. Liu, L.; He, N.; Wu, T.; Hu, P.; Tong, G. Co/C/Fe/C Hierarchical Flowers with Strawberry-like Surface as Surface Plasmon for Enhanced Permittivity, Permeability, and Microwave Absorption Properties. Chem. Eng.J. 2019, 355, 103-108.

6. Zhou, C.; Wu, C.; Yan, M. A Versatile Strategy towards Magnetic/Dielectric Porous Heterostructure with Confinement Effect for Lightweight and Broadband Electromagnetic Wave Absorption. Chem. Eng.J. 2019, 370, 988-996.

7. Wang, Y.; Han, X.; Xu, P.; Liu, D.; Cui, L.; Zhao, H.; Du, Y. Synthesis of Pomegranate-like $\mathrm{Mo}_{2} \mathrm{C} @ \mathrm{C}$ Nanospheres for Highly Efficient Microwave Absorption. Chem. Eng.J. 2019, 372, 312-320.

8. Ge, C.; Wang, L.; Liu, G.; Chen, H. Synthesis and Electromagnetic Absorption Properties of $\mathrm{CeO}_{2} @$ Fe Composites with Core-shell Structure. J. Magn. Magn. Mater. 2019, 485, $228-235$.

9. Ning, M. Q.; Lu, M. M.; Li, J. B.; Chen, Z.; Dou, Y. K.; Wang, C. Z.; Rehman, F.; Cao, M. S.; Jin, H. B. Two-dimensional Nanosheets of $\mathrm{MoS}_{2}$ : a Promising Material with High Dielectric Properties and Microwave Absorption Performance. Nanoscale 2015, 7 (38), 15734-14740.

10. Li, Y.; Liu, X.; Nie, X.; Yang, W.; Wang, Y.; Yu, R.; Shui, J. Multifunctional OrganicInorganic Hybrid Aerogel for Self-Cleaning, Heat-Insulating, and Highly Efficient Microwave Absorbing Material. Adv. Funct. Mater. 2019, 29 (10), 1807624 (1-9).

11. Yin, Y.; Liu, X.; Wei, X.; Li, Y.; Nie, X.; Yu, R.; Shui, J. Magnetically Aligned Co- 
C/MWCNTs Composite Derived from MWCNT-Interconnected Zeolitic Imidazolate Frameworks for a Lightweight and Highly Efficient Electromagnetic Wave Absorber. ACS Appl. Mater. Interfaces 2017, 9 (36), 30850-30861.

12. Cheng, Y.; Zhao, Y.; Zhao, H.; Lv, H.; Qi, X.; Cao, J.; Ji, G.; Du, Y. Engineering Morphology Configurations of Hierarchical Flower-like $\mathrm{MoSe}_{2}$ Spheres Enable Excellent Low-frequency and Selective Microwave Response Properties. Chem. Eng. J. 2019, 372, 390-398.

13. Biswas, S.; Dutta, S.; Panja, S. S.; Bose, S. Template-Free Synthesis of "Wool-Ball"-Like Hollow CuS Structures can Effectively Suppress Electromagnetic Radiation: A Mechanistic Insight. J. Phys. Chem. C 2019, 123 (28), 17136-17147.

14. Huang, T.; He, M.; Zhou, Y.; Li, S.; Ding, B.; Pan, W.; Huang, S.; Tong, Y. Solvothermal Synthesis of Flower-like CoS Hollow Microspheres with Excellent Microwave Absorption Properties. RSC Adv. 2016, 6 (102), 100392-100400.

15. Zhou, S.; Huang, Y.; Xu, L.; Zheng, W. Microwave-assisted Synthesis of Graphene$\mathrm{NiS} / \mathrm{Ni}_{3} \mathrm{~S}_{2}$ Composites for Enhanced Microwave Absorption Behaviors through a Sulfuration Method. Ceram. Int. 2018, 44 (17), 21786-21793.

16. Cheng, Y.; Guo, Y.; Zhang, Z.; Dong, S.; Wang, H. Facile Synthesis of $\mathrm{Ni}_{\mathrm{x}} \mathrm{Co}_{3-\mathrm{x}} \mathrm{S}_{4}$ Hollow Nanoprism with Broader Electromagnetic Absorption Properties: Effect of Ni/Co Atomic Ratios. J. Alloy. Compd. 2018, 767, 323-329.

17. Sun, Y. C.; Li, D. P.; Yang, Y.; Fan, L. S.; Wu, S.; Wang, P.; Song, Y., Achieving Rough Sphere-shaped $\mathrm{ZnS}$ with Superior Attenuation Electromagnetic Absorption Performance. RSC Adv. 2017, 7 (7), 3907-3913.

18. Liu, Y.; Wu, Y.; Li, K.; Wang, J.; Zhang, C.; Ji, J.; Wang, W., Amorphous SnS Nanosheets/Graphene Oxide Hybrid with Efficient Dielectric Loss to Improve the HighFrequency Electromagnetic Wave Absorption Properties. Appl. Surf. Sci. 2019, 486, 344-353.

19. Lv, H.; Yang, Z.; Wang, P. L.; Ji, G.; Song, J.; Zheng, L.; Zeng, H.; Xu, Z. J. A VoltageBoosting Strategy Enabling a Low-Frequency, Flexible Electromagnetic Wave Absorption Device. Adv. Mater. 2018, 30 (15), 1706343 (1-8).

20. Liu, W.; Tan, S.; Yang, Z.; Ji, G. Hollow Graphite Spheres Embedded in Porous Amorphous Carbon Matrices as Lightweight and Low-Frequency Microwave Absorbing Material through Modulating Dielectric Loss. Carbon 2018, 138, 143-153.

21. Yang, T.; Liu, Y.; Yang, D.; Deng, B.; Huang, Z.; Ling, C. D.; Liu, H.; Wang, G.; Guo, Z.; Zheng, R. Bimetallic Metal-Organic Frameworks Derived Ni-Co-Se@C Hierarchical Bundlelike Nanostructures with High-Rate Pseudocapacitive Lithium Ion Storage. Energy Storage Mater. 2019, 17, 374-384.

22. Feng, C.; Zhao, G.; Li, Y.; Cheng, H.; Wang, Z. S. Single-Crystal Cobalt Selenide Nanobelt as a Highly Efficient Cathode for Stable Quasi-solid-state Dye Sensitized Solar Cell. J. Power Sources 2019, 426, 16-22.

23. Yue, H.; Yu, B.; Qi, F.; Zhou, J.; Wang, X.; Zheng, B.; Zhang, W.; Li, Y.; Chen, Y. Interwoven $\mathrm{CoSe}_{2} / \mathrm{CNTs}$ Hybrid as a Highly Efficient and Stable Electrocatalyst for Hydrogen Evolution Reaction. Electrochim. Acta 2017, 253, 200-207.

24. Li, H.; Qian, X.; Zhu, C.; Jiang, X.; Shao, L.; Hou, L. Template Synthesis of $\mathrm{CoSe}_{2} / \mathrm{Co}_{3} \mathrm{Se}_{4}$ Nanotubes: Tuning of Their Crystal Structures for Photovoltaics and Hydrogen Evolution in Alkaline Medium. J. Mater. Chem. A 2017, 5 (9), 4513-4526.

25. Zhang, Y.; Pan, A.; Wang, Y.; Cao, X.; Zhou, Z.; Zhu, T.; Liang, S.; Cao, G. Self-Templated Synthesis of N-doped $\mathrm{CoSe}_{2} / \mathrm{C}$ Double-Shelled Dodecahedra for High-Performance Supercapacitors. Energy Storage Mater. 2017, 8, 28-34. 
26. Carvalho-Jr, W. M.; Mendonça-Ferreira, L.; Costa, F. N.; Ferreira, F. F.; Muche, D. N. F.; Tofanello, R. A.; Castro, R. H. R.; Souza, F. L. Annealing Control of Hydrothermally Grown Hematite Nanorods: Implication of Structural Changes and $\mathrm{Cl}$ Concentration on Weak Ferromagnetism. J. Alloy. Compd. 2019, 799, 83-88.

27. Jian, X.; Xiao, X.; Deng, L.; Tian, W.; Wang, X.; Mahmood, N.; Dou, S. Heterostructured Nanorings of $\mathrm{Fe}_{-} \mathrm{Fe}_{3} \mathrm{O}_{4} @ \mathrm{C}$ Hybrid with Enhanced Microwave Absorption Performance. ACS Appl. Mater. Interfaces 2018, 10 (11), 9369-9378.

28. Hosseini, H.; Shahrokhian, S. Self-Supported Nanoporous Zn-Ni-Co/Cu Selenides Microball Arrays for Hybrid Energy Storage and Electrocatalytic Water/Urea Splitting. Chem. Eng. J. 2019, 375, 122090 (1-19).

29. Chiu, I. T.; Li, C. T.; Lee, C. P.; Chen, P. Y.; Tseng, Y. H.; Vittal, R.; Ho, K. C. NanoclimbingWall-Like $\mathrm{CoSe}_{2} /$ Carbon Composite Film for the Counter Electrode of a Highly Efficient DyeSensitized Solar Cell: A Study on the Morphology Control. Nano Energy 2016, 22, 594-606.

30. Jian, X.; Wang, H.; Rao, G.; Jiang, L.; Wang, H.; Subramaniyam, C. M.; Mahmood, A.; Zhang, W.; Xiang, Y.; Dou, S. X.; Zhou, Z.; Hui, D.; Kalantar-Zadeh, K.; Mahmood, N. SelfTunable Ultrathin Carbon Nanocups as the Electrode Material of Sodium-Ion Batteries with Unprecedented Capacity and Stability. Chem. Eng. J. 2019, 364, 578-588.

31. Li, Y.; Li, S.; Zhang, T.; Shi, L.; Liu, S.; Zhao, Y. 3D Hierarchical $\mathrm{Co}_{3} \mathrm{O}_{4} /$ Reduced Graphene Oxide/Melamine Derived Carbon Foam as a Comprehensive Microwave Absorbing Material. $J$. Alloy. Compd. 2019, 792, 424-431.

32. Yang, T.; Yang, D.; Liu, Y.; Liu, J.; Chen, Y.; Bao, L.; Lu, X.; Xiong, Q.; Qin, H.; Ji, Z.; Ling, C. D.; Zheng, R. MOF-Derived Carbon-Encapsulated Cobalt Sulfides Orostachys-Like Micro/Nano-Structures as Advanced Anode Material for Lithium Ion Batteries. Electrochim. Acta 2018, 290, 193-202.

33. Gao, J.; Liu, L.; Qiu, H. J.; Wang, Y., Engineering Phase Transformation of Cobalt Selenide in Carbon Cages and the Phases' Bifunctional Electrocatalytic Activity for Water Splitting. Nanotechnology 2017, 28 (31), 315401 (1-9).

34. Yuan, M.; Wang, M.; Lu, P.; Sun, Y.; Dipazir, S.; Zhang, J.; Li, S.; Zhang, G. Tuning Carbon Nanotube-Grafted Core-Shell-Structured Cobalt Selenide@Carbon Hybrids for Efficient Oxygen Evolution Reaction. J. Colloid Interface Sci. 2019, 533, 503-512.

35. Liu, P.; Zhang, Y.; Yan, J.; Huang, Y.; Xia, L.; Guang, Z. Synthesis of Lightweight N-doped Graphene Foams with Open Reticular Structure for High-Efficiency Electromagnetic Wave Absorption. Chem. Eng. J. 2019, 368, 285-298.

36. Jian, X.; Tian, W.; Li, J.; Deng, L.; Zhou, Z.; Zhang, L.; Lu, H.; Yin, L.; Mahmood, N. HighTemperature Oxidation-Resistant $\mathrm{ZrN}_{0.4} \mathrm{~B}_{0.6} / \mathrm{SiC}$ Nanohybrid for Enhanced Microwave Absorption. ACS Appl. Mater. Interfaces 2019, 11 (17), 15869-15880.

37. Yang, H. J.; Cao, W. Q.; Zhang, D. Q.; Su, T. J.; Shi, H. L.; Wang, W. Z.; Yuan, J.; Cao, M. S. $\mathrm{NiO}$ Hierarchical Nanorings on SiC: Enhancing Relaxation to Tune Microwave Absorption at Elevated Temperature. ACS Appl. Mater. Interfaces 2015, 7 (13), 7073-7087.

38. Han, M.; Yin, X.; Ren, S.; Duan, W.; Zhang, L.; Cheng, L. Core/Shell Structured C/ZnO Nanoparticles Composites for Effective Electromagnetic Wave Absorption. RSC Adv. 2016, 6 (8), 6467-6474.

39. Qiang, R.; Du, Y.; Zhao, H.; Wang, Y.; Tian, C.; Li, Z.; Han, X.; Xu, P. Metal Organic Framework-Derived Fe/C Nanocubes toward Efficient Microwave Absorption. J. Mater. Chem. A 2015, 3 (25), 13426-13434.

40. Mu, C.; Du, X.; Nie, A.; Wang, B.; Wen, F.; Xiang, J.; Zhai, K.; Liu, Z. Microwave 
Absorption Properties of Heterostructure Composites of Two Dimensional Layered Magnetic Materials and Graphene Nanosheets. Appl. Phys. Lett. 2019, 115 (4), 043103 (1-5).

41. Dong, X. L.; Zhang, X. F.; Huang, H.; Zuo, F. Enhanced Microwave Absorption in Ni/Polyaniline Nanocomposites by Dual Dielectric Relaxations. Appl. Phys. Lett. 2008, 92 (1), $013127(1-3)$.

42. Song, Z.; Liu, X.; Sun, X.; Li, Y.; Nie, X.; Tang, W.; Yu, R.; Shui, J. Alginate-Templated Synthesis of CoFe/carbon Fiber Composite and the Effect of Hierarchically Porous Structure on Electromagnetic Wave Absorption Performance. Carbon 2019, 151, 36-45.

43. Xia, Z.; He, J.; Ou, X.; Li, Y.; Wang, Y.; Shen, B.; Yu, X.; He, S.; Zhao, D.; Yu, G. Enhanced Microwave-Absorption Performance of FeCoB/Polyimide-Graphene Composite by Electric Field Modulation. Compos. Sci. Technol. 2017, 152, 222-230.

44. Sun, G.; Wu, H.; Liao, Q.; Zhang, Y. Enhanced Microwave Absorption Performance of Highly Dispersed CoNi Nanostructures Arrayed on Graphene. Nano Res. 2018, 11 (5), 2689-2704.

45. Liu, D.; Du, Y.; Li, Z.; Wang, Y.; Xu, P.; Zhao, H.; Wang, F.; Li, C.; Han, X. Facile Synthesis of 3D Flower-like Ni Microspheres with Enhanced Microwave Absorption Properties. J. Mater. Chem. C 2018, 6 (36), 9615-9623.

46. Yu, X.; Wang, L.; Liu, J.; Xue, S.; Yang, L.; Li, X.; Zhang, J.; Xing, L.; Chen, G.; Wang, M.; Che, R. Ferromagnetic $\mathrm{Co}_{20} \mathrm{Ni}_{80}$ Nanoparticles Encapsulated Inside Reduced Graphene Oxide Layers with Superior Microwave Absorption Performance. J. Mater. Chem. C 2019, 7 (10), 2943-2953.

47. Jian, X.; Wu, B.; Wei, Y.; Dou, S. X.; Wang, X.; He, W.; Mahmood, N. Facile Synthesis of $\mathrm{Fe}_{3} \mathrm{O}_{4} / \mathrm{GCs}$ Composites and Their Enhanced Microwave Absorption Properties. ACS Appl. Mater. Interfaces 2016, 8 (9), 6101-6109.

48. Wang, J.; Or, S. W.; Tan, J. Enhanced Microwave Electromagnetic Properties of Core/Shell/Shell-Structured $\mathrm{Ni} / \mathrm{SiO}_{2} /$ Polyaniline Hexagonal Nanoflake Composites with Preferred Magnetization and Polarization Orientations. Mater. Design 2018, 153, 190-202.

49. Liu, T.; Pang, Y.; Zhu, M.; Kobayashi, S. Microporous Co@CoO Nanoparticles with Superior Microwave Absorption Properties. Nanoscale 2014, 6 (4), 2447-2454.

50. Zhang, Y.; Piao, M.; Zhang, H.; Zhang, F.; Chu, J.; Wang, X.; Shi, H.; Li, C. Synthesis of Mesoporous Hexagonal Cobalt Nanosheets with Low Permittivity for Enhancing Microwave Absorption Performances. J. Magn. Magn. Mater. 2019, 486, 165272 (1-10).

51. Wu, G.; Zhang, H.; Luo, X.; Yang, L.; Lv, H. Investigation and Optimization of Fe/ $\mathrm{ZnFe}_{2} \mathrm{O}_{4}$ as a Wide-Band Electromagnetic Absorber. J. Colloid Interface Sci. 2019, 536, 548-555.

52. Zhang, Y.; Zhang, H. B.; Wu, X.; Deng, Z.; Zhou, E.; Yu, Z. Z. Nanolayered Cobalt@Carbon Hybrids Derived from Metal-Organic Frameworks for Microwave Absorption. ACS Appl. Nano Mater. 2019, 2 (4), 2325-2335.

53. Deng, R.; Chen, B.; Li, H.; Zhang, K.; Zhang, T.; Yu, Y.; Song, L. MXene/Co ${ }_{3} \mathrm{O}_{4}$ Composite Material: Stable Synthesis and its Enhanced Broadband Microwave Absorption. Appl. Surf. Sci. 2019, 488, 921-930.

54. Ramasubramaniam, R.; Chen, J.; Liu, H. Homogeneous Carbon Nanotube/Polymer Composites for Electrical Applications. Appl. Phys. Lett. 2003, 83 (14), 2928-2930.

55. Naito, Y.; Suetake, K. Application of Ferrite to Electromagnetic Wave Absorber and its Characteristics. IEEE T Microw. Theory 1971, MTS-19, 66-72.

56. Liu, P.; Yao, Z.; Zhou, J.; Yang, Z.; Kong, L. B. Small Magnetic Co-doped NiZn ferrite/Graphene Nanocomposites and Their Dual-Region Microwave Absorption Performance. $J$. 
Mater. Chem. C 2016, 4 (41), 9738-9749.

57. Lu, B.; Huang, H.; Dong, X. L.; Zhang, X. F.; Lei, J. P.; Sun, J. P.; Dong, C. Influence of Alloy Components on Electromagnetic Characteristics of Core/Shell-Type Fe-Ni Nanoparticles. J. Appl. Phys. 2008, 104 (11), 114313 (1-6).

58. Guo, Y.; Zhang, X.; Feng, X.; Jian, X.; Zhang, L.; Deng, L. Non-Isothermal Oxidation Kinetics of FeSiAl Alloy Powder for Microwave Absorption at High Temperature. Compos. Part B-Eng. 2018, 155, 282-287.

59. Rehman, S.; Wang, J.; Luo, Q.; Sun, M.; Jiang, L.; Han, Q.; Liu, J.; Bi, H. Starfish-like $\mathrm{C} / \mathrm{CoNiO}_{2}$ Heterostructure Derived from ZIF-67 with Tunable Microwave Absorption Properties. Chem. Eng. J. 2019, 373, 122-130.

60. Zhang, X. J.; Zhu, J. Q.; Yin, P. G.; Guo, A. P.; Huang, A. P.; Guo, L.; Wang, G. S. Tunable High-Performance Microwave Absorption of $\mathrm{Co}_{1-\mathrm{x}} \mathrm{S}$ Hollow Spheres Constructed by Nanosheets within Ultralow Filler Loading. Adv. Funct. Mater. 2018, 28 (49), 1800761 (1-7).

61. Li, J.; Zhao, X.; Liu, J.; Zhang, L.; Yang, C. Ultralight Carbon-Based $\mathrm{Co}(\mathrm{OH})_{2}-\mathrm{Co}_{3} \mathrm{O}_{4} /$ Nanocomposite with Superior Performance in Wave Absorption. J. Alloy. Compd. 2019, 777 , 954-962.

62. Gu, W.; Quan, B.; Liang, X.; Liu, W.; Ji, G.; Du, Y. Composition and Structure Design of $\mathrm{Co}_{3} \mathrm{O}_{4}$ Nanowires Network by Nickel Foam with Effective Electromagnetic Performance in C and X Band. ACS Sustain. Chem. Eng. 2019, 7 (5), 5543-5552.

63. Xiao, X.; Zhu, W.; Tan, Z.; Tian, W.; Guo, Y.; Wang, H.; Fu, J.; Jian, X. Ultra-Small $\mathrm{Co} / \mathrm{CNTs}$ Nanohybrid from Metal Organic Framework with Highly Efficient Microwave Absorption. Compos. Part B-Eng. 2018, 152, 316-323.

64. Su, X.; Wang, D.; Yan, Q.; Guo, X.; Fang, S.; Liu, Y. Preparation, Microstructure, Electromagnetic Property and Microwave Absorption Property of $\mathrm{MoS}_{2}$ Nanopowder by Hydrothermal Method at Various Reaction Temperatures. J. Mater. Sci. 2017, 29 (5), 4020-4029. 65. Deng, J.; Zhang, X.; Zhao, B.; Bai, Z.; Wen, S.; Li, S.; Li, S.; Yang, J.; Zhang, R. Fluffy Microrods to Heighten the Microwave Absorption Properties through Tuning the Electronic State of Co/CoO. J. Mater. Chem. C 2018, 6 (26), 7128-7140.

66. Zhao, B.; Shao, G.; Fan, B.; Zhao, W.; Chen, Y.; Zhang, R., Facile Synthesis of Crumpled ZnS Net-Wrapped Ni Walnut Spheres with Enhanced Microwave Absorption Properties. RSC Adv. 2015, 5 (13), 9806-9814.

67. Guan, X. H.; Qu, P.; Guan, X.; Wang, G. S., Hydrothermal Synthesis of Hierarchical $\mathrm{CuS} / \mathrm{ZnS}$ Nanocomposites and Their Photocatalytic and Microwave Absorption Properties. RSC Adv. 2014, 4 (30), 15579-15585. 